\title{
RESEARCH
}

Open Access

\section{LSH interacts with and stabilizes GINS4 transcript that promotes tumourigenesis in non-small cell lung cancer}

Rui Yang ${ }^{1,2+}, \mathrm{Na} \mathrm{Liu}^{1,2+}$, Ling Chen ${ }^{1,2}$, Yiqun Jiang ${ }^{1,2}$, Ying Shi ${ }^{1,2}$, Chao Mao ${ }^{1,2}$, Yating Liu ${ }^{1,2}$, Min Wang ${ }^{1,2}$, Weiwei Lai ${ }^{1,2}$, Haosheng Tang ${ }^{1,2}$, Menghui Gao ${ }^{1,2}$, Desheng Xiao ${ }^{3}$, Xiang Wang ${ }^{4}$, Fenglei Y ${ }^{4}$, Ya Cao ${ }^{1,2}$, Qin Yan ${ }^{5^{*}}$, Shuang Liu ${ }^{6^{*}}$ and Yongguang Tao ${ }^{1,2,4^{*}}$ (D)

\begin{abstract}
Background: Elucidating mechanisms in oncogenes and epigenetic modifiers are needed to gain insights into the etiology and treatment of cancer, regulation of oncogene by chromatin modifiers at post-transcriptional level is critical and remains unclear. We have investigated the role of GINS4 in NSCLC.

Methods: The expression of chromatin modifier lymphoid-specific helicase (LSH) and GINS4 was assessed in tumor and normal tissue from 79 patients with NSCLC with clinical characteristics. HBE, A549, H358, and H522, PC9, 95C and 95D were cultured after overexpression or silencing of GIAT4RA. Cell proliferation assay, cell migration and invasion assays, plate colony formation assay, immunofluorescence assay, Operetta ${ }^{\circledR}$ high-content screening and analysis, Western blot analysis and Co-Immunoprecipitation (Co-IP) assay, RNA immunoprecipitation assay and tumor growth assay was used to address the potential interplay of between GINS4 and LSH, and the functional of GINS4.

Results: GINS4 is highly expressed in lung cancer cells and tissues, and GINS4 expression is not association with clinical risk factors, but linked with clinical stage and lymphatic metastasis status. Higher expression of GINS4 poorly linked with overall survival in lung adenocarcinomas. Furthermore, GINS4 promoted many characteristics of tumorigenesis including cell growth, clonal formation, migration and invasion, epithelial-mesenchymal transition, tumor sphere and tumor growth in vivo. Interestingly, our results demonstrated that LSH increases GINS4 expression through binding to 3'UTR region of GINS4 and stabilizing its mRNA levels. Finally, LSH overexpression rescues GINS4 knockdown-induced features.
\end{abstract}

Conclusions: GINS4 facilitates lung cancer progression by promoting key characteristics of tumor potential, and LSH epigenetically interacts with and stabilizes GINS4 transcripts.

Keywords: GINS4, LSH, mRNA stability, Lung cancer

\footnotetext{
*Correspondence: taoyong@csu.edu.cn; shuangliu2016@csu.edu.cn; qin.yan@yale.edu

${ }^{\dagger}$ Rui Yang and Na Liu contributed equally to this work.

${ }^{5}$ Department of Pathology, Yale School of Medicine, New Haven, CT 06520,

USA

${ }^{6}$ Department of Oncology, Institute of Medical Sciences, Xiangya Hospital,

Central South University, Changsha 410008, Hunan, China

${ }^{1}$ Department of Pathology, Key Laboratory of Carcinogenesis and Cancer

Invasion, Ministry of Education, Xiangya Hospital, Central South University,

Changsha 410078, Hunan, China

Full list of author information is available at the end of the article
}

(c) The Author(s). 2019 Open Access This article is distributed under the terms of the Creative Commons Attribution 4.0 International License (http://creativecommons.org/licenses/by/4.0/), which permits unrestricted use, distribution, and reproduction in any medium, provided you give appropriate credit to the original author(s) and the source, provide a link to the Creative Commons license, and indicate if changes were made. The Creative Commons Public Domain Dedication waiver (http://creativecommons.org/publicdomain/zero/1.0/) applies to the data made available in this article, unless otherwise stated. 


\section{Background}

Lung cancer is classified into small cell lung cancer and non-small cell lung cancer (NSCLC), including adenocarcinomas (ADC) and squamous cell carcinoma (SCC), which account for $80-85 \%$ of all lung cancer cases [1]. Lung cancer is a leading cause of cancer-related deaths and the most common cancer worldwide, including China, accounting for $>2.8$ million deaths in 2015 [2]. Epigenetic modifiers, including chromatin remodeling factors, play important roles in cancer development and progression [1, $3]$. Although studies performed on oncogenes and cancer epigenetic factors over the last decade have identified numerous epigenetic modifiers that are involved in the progression of various cancers [4-8], mechanisms underlying the interplay between epigenetic factors and oncogenes in lung cancer remain unclear.

Chromatin modifiers display widespread interactions with RNA transcripts, and regulation of the length of the $3^{\prime}$ UTR (3'-untranslated region) is suggested to be associated with the ability of chromatin modifiers to interact with both chromatin and mRNA transcripts [9-12]. SWI/SNF is a large multi-subunit chromatin remodeling complex that can be combinatorially assembled to yield hundreds to thousands of biochemically unique complex and are frequently disrupted in human cancer [13, 14]. Lymphoidspecific helicase (LSH), which belongs to SNF2 family of chromatin-remodeling ATPases, is critical for the normal development of plants and mammals because it establishes correct DNA methylation levels and patterns [15-19]. LSH maintains genome stability in mammalian somatic cells $[20,21]$; Moreover, LSH contributes to the malignant progression [22-25]. Our recent study indicated that lncRNA HOTAIR interacts and forms an intact complex with LSH to affect the expression of target genes [26].

Go, Ichi, Nii, and San (means five, one, two, and three, respectively, in Japanese) complex subunit 4 (GINS4) is a member of GINS family of proteins that are essential for the initiation of DNA replication in yeast and Xenopus egg extracts [27, 28]. GINS4 is involved in early embryogenesis in mice and maintains cell cycle progression and genome integrity in Drosophila [28, 29], suggesting its role in tumorigenesis. However, the relevance of GINS4 in lung cancer has not been determined to date.

In this study, we examined the physiological role of GINS4 in lung cancer progression and their potential epigenetic mechanisms. We found that LSH increased GINS4 expression by stabilizing its mRNA level posttranscriptionally.

\section{Material and methods}

Cell culture, antibodies, plasmids, shRNAs and chemicals Normal lung cell lines, HBE (ATCC: CRL-2741 $1^{\mathrm{m}}$ ) were purchased from the ATCC. The lung cancer cell lines A549 (ATCC: CCL-185 $5^{\mathrm{mm}}$ ), H358 (ATCC: CRL-5807 $7^{\mathrm{mm}}$ ), and
H522 (ATCC: CRL-5810 The lung cancer cell lines PC9, 95C and 95D were obtained from the Cancer Research Institute of Central South University. A549 cells were maintained in DME/ F12 1:1(Hyclone), $293 \mathrm{~T}$ cells were maintained in DMEM (Gibco), and the other cells were maintained in RPMI 1640 (Gibco). All media were supplemented with $10 \%$ (v/ v) FBS, and all the cells were maintained at $37^{\circ} \mathrm{C}$ in an atmosphere of $5 \% \mathrm{CO}_{2}$. All the cell lines yielded negative result for mycoplasma contamination. All the cell lines were passaged $<10$ times after their initial revival from frozen stocks and were authenticated by performing short tandem repeat profiling before their use.

Actinomycin D, MG132, and CHX were purchased from Selleck (Houston, TX). Vectors overexpressing truncated FLAG-LSH fragments were generated by cloning cDNAs encoding these fragments into pLVX-EF1 $\alpha$-IRES-Puro vector (catalog no. 631988; Clontech, Mountain View, CA) by using restriction enzymes EcoRI and BamHI (Takara). Lentiviral vectors expressing GINS4 were purchased from Vigene Biosciences (http://www.vigenebio.com; Shandong, China).

Lentiviral shRNA vectors targeting human GINS4, and $L S H$ and a non-targeting control vector were purchased from Genechem (http://www.genechem. com.cn; Shanghai, China). All the plasmid vectors were verified by performing sequencing.

\section{Western blot analysis}

Western blotting analysis was performed as described previously [30]. Primary antibodies against LSH and $\alpha-$ tubulin were purchased from Santa Cruz Biotechnology, and primary antibody against GINS4 was purchased from GeneTex. EMT Antibody Sampler Kit and primary antibodies against histone $\mathrm{H} 3$ were purchased from Cell Signaling Technology, and primary antibody against $\beta$-actin was purchased from Sigma-Aldrich (St. Louis, MO).

\section{Immunohistochemistry (IHC) analysis}

Lung cancer tissue samples, which were validated by pathologist Dr. Desheng Xiao (Xiangya Hospital), were obtained from the Department of Pathology of Xiangya Hospital. A lung cancer tissue array was purchased from Pantomics (Richmond, CA). IHC analysis of paraffinembedded tissue samples obtained from patients with lung cancer was performed as described previously [31].

\section{Quantitative reverse transcription-PCR and RNA immunoprecipitation assay}

qRT-PCR was performed as described previously [30, 31]. Primer sequences used for performing qRT-PCR are as follows: GINS4 forward, 5'-TCAAGCCTGTAATCCCAGC A-3'; GINS4 reverse, 5'-GTTCAAGCGATTCTCCTGCC3'; $\beta$-actin forward, 5'-CACCATTGGCAATGAGCGGTT 
C-3'; and $\beta$-actin reverse, $5^{\prime}$-AGGTCTTTGCGGATGTCCACGT-3'. Results are expressed as mean \pm SD of three independent experiments.

RNA immunoprecipitation assay was performed as described previously [32], a total of $10^{7}$ cells were harvested by trypsinization and resuspended in $2 \mathrm{~mL}$ of PBS. The cell lysate was pelleted by centrifugation at $4{ }^{\circ} \mathrm{C}$ and $500 \times g$ for $15 \mathrm{~min}$. The cell lysate was resuspended in $1 \mathrm{~mL}$ of RIP buffer, split into three fractions (for Input, Mock, and IP), and then centrifuged at $4{ }^{\circ} \mathrm{C}$ and $13,000 \mathrm{rpm}$ for $10 \mathrm{~min}$. Antibodies against normal mouse IgG (Merck Millipore, catalog no. 12-371), normal rabbit IgG (Cell Signaling Technology, catalog no. 2729), and anti-FLAG M2 Magnetic Beads (Sigma Aldrich, catalog no. M8823) were added to the supernatant and incubated overnight at $4{ }^{\circ} \mathrm{C}$ with gentle rotation. Next, $40 \mu \mathrm{L}$ of protein A/G beads were added and the mixture was incubated at $4{ }^{\circ} \mathrm{C}$ for an additional hour. The beads were pelleted at $2500 \mathrm{rpm}$ for $30 \mathrm{~s}$, washed three times with $500 \mu \mathrm{L}$ of RIP buffer and one time with PBS, and then resuspended in $1 \mathrm{~mL}$ of RNAiso Plus. The total RNA (input control) and RNA precipitated with the isotype control (IgG) for each antibody were assayed simultaneously with all test samples. The coprecipitated RNAs were detected by qRT-PCR for GINS4.

\section{Cell proliferation assay, cell migration and invasion assays, plate colony formation assay, immunofluorescence assay, and operetta ${ }^{\circledR}$ high-content screening and analysis}

These assays were performed as described previously [30, 31]. Detailed protocols of these assays are mentioned in Additional file 1 Supplementary Material and Methods.

\section{Oncosphere formation assay}

These assays were performed as described previously [33, 34]. Cells were seeded on ultra-low attachment culture dishes (Corning, Corning, NY) in serum-free DMEM-F12 medium containing $50 \mu \mathrm{g} / \mathrm{ml}$ insulin (Sigma-Aldrich St. Louis, MO), 0.4\% Albumin Bovine Fraction V (Sigma-Aldrich St. Louis, MO), $\mathrm{N}^{-2}$ Plus Media Supplement (Life Technologies, Grand Island, NY), B-27 Supplement (Life Technologies, Grand Island, NY), $20 \mu \mathrm{g} / \mathrm{ml}$ EGF (PeproTech Rocky Hill, NJ), and $10 \mu \mathrm{g} / \mathrm{ml}$ basic FGF (PeproTech, Rocky Hill, NJ) to support the growth of undifferentiated oncospheres. Cells were incubated in a $\mathrm{CO} 2$ incubator for 1-2 weeks, and the numbers of oncosphere cells were counted under a microscope.

\section{Luciferase assay}

To test the regulation of LSH on the GINS4 3'UTR, we constructed a pMiR-REPORT luciferase vector expressing the 3'UTR of GINS4. Luciferase levels were normalized to those of a non-responsive vector expressing Renilla luciferase.

\section{Nude mice and study approval}

The xenograft tumor formation assay was essentially performed as previously described [25, 32-35]. All procedures for animal study were approved by the Institutional Animal Care and Use Committee of the Xiangya School of Medicine of Central South University and confirmed to the legal mandates and federal guidelines for the care and maintenance of laboratory animals. Fourweek-old male $\mathrm{BALB} / \mathrm{c}$ athymic mice were purchased from the Hunan SJA Laboratory Animal Co. Ltd. (http:// www.hnsja.com) and housed in dedicated pathogen-free barrier facilities. The mice were injected with the indicated cells in the mammary fat pad. Injected mice were imaged from both the dorsal and ventral sides every 3 days. Data were analyzed using Student's t-test; a $p$ value $<0.05$ was considered significant.

\section{Reproducibility and statistics}

All experiments performed in this study were repeated at least three times. Results of western blotting analysis are representative of three independent experiments. All experiments, except experiments involving nude mice, were repeated at least three times.

Results are expressed as mean \pm SD or SEM, as indicated. A two-tailed Student's t-test was used for performing intergroup comparisons. A $p$ value of $<0.05$ was considered statistically significant. ", ***, and ${ }^{* * *}$ indicate $P<0.05, P<0.01$, and $P<0.001$, respectively.

\section{Results}

GINS4 is highly expressed in lung cancer tissues and is associated with the poor survival of patients with lung $A D C$

We previously found that LSH upregulated GINS4 mRNA expression using RNA sequencing [36]. Here we first determined the role of GINS4 in patients with lung cancer, we performed qRT-PCR analysis of an independent panel of 79 primary NSCLC tissues and normal lung tissues. GINS4 expression was upregulated in the primary lung cancer tissues (Fig. 1a). To further determine GINS4 expression level in lung cancer, we performed IHC analysis of tissues obtained from patients with lung cancer. GINS4 protein was detected in the cytoplasm and nucleus of cells isolated from normal lung tissues, and its expression was highly increased in both lung ADC and SCC tissues (Fig. 1b-c). Furthermore, TCGA database indicated that GINS4 was highly expressed in both lung ADC and SCC tissues (Additional file 1: Figure S1A-B). Moreover, GINS4 protein levels were clearly increased in lung cancer tissues obtained from cases 1-10 in Fig. 1d with lung ADCs, and cases 11-18 of Fig. 1d with lung SCCs.

Finally, multivariate analysis showed that the expression level of GINS4 was independent of clinical risk factors 


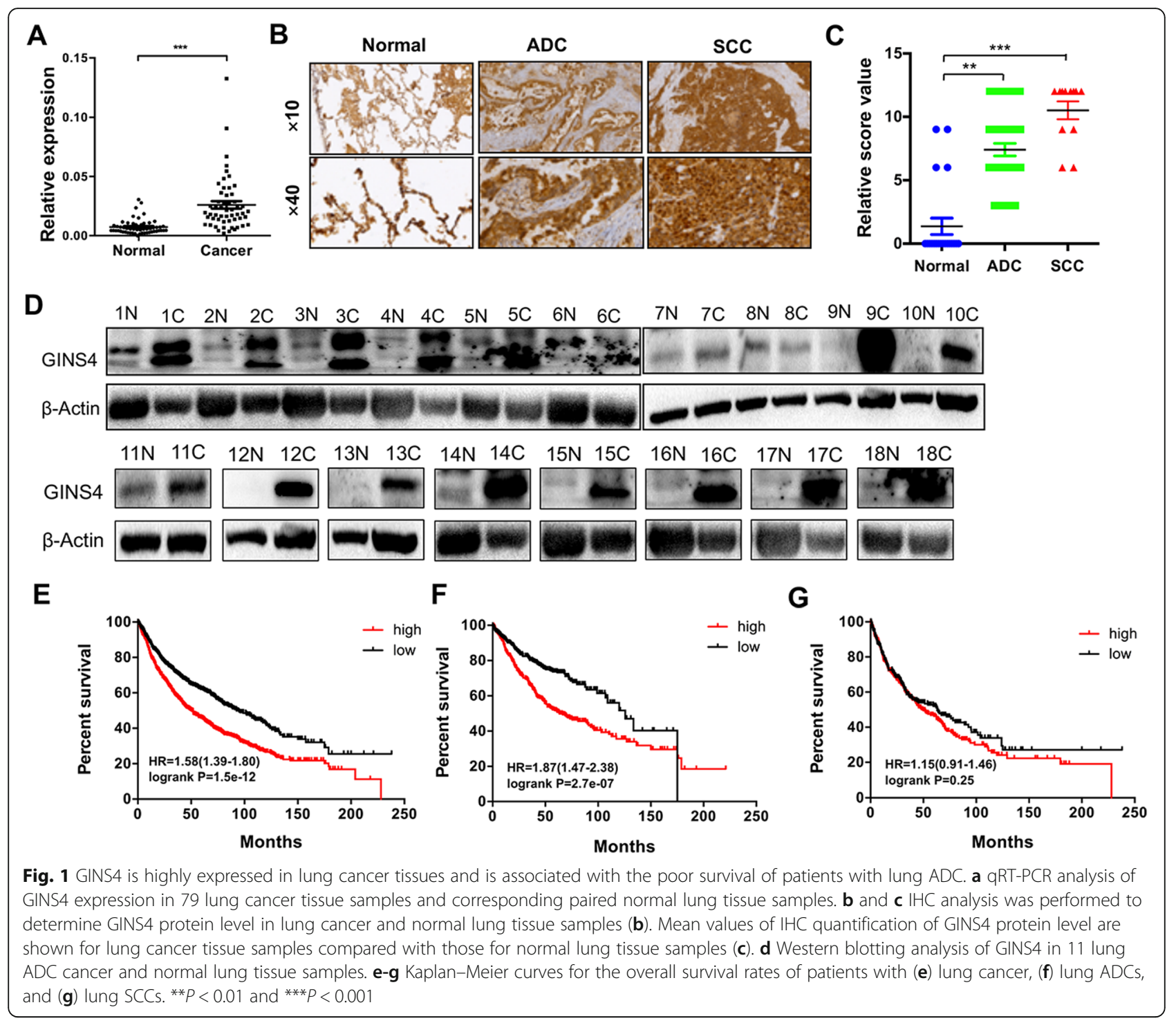

such as gender, smoking, tumor differentiation and tumor size, but linked with clinical stages and lymphatic metastasis (Table 1). Kaplan-Meier analysis of these patients showed that GINS4 expression was associated with poor overall survival in all lung cancers (Fig. 1e), and lung ADCs (Fig. 1f), but not in lung SCCs (Fig. 1g). Together, these findings indicate that GINS4 is highly expressed in lung cancer and suggest that it functions as an oncogene in lung cancer progression.

\section{LSH induces GINS4 expression and binds with GINS4 transcript}

Next, we performed western blotting analysis to show that LSH overexpression promoted GINS4 protein expression in H358, PC9 and HBE cells (Fig. 2a-b). In addition, stable LSH knockdown decreased GINS4 protein expression in A549 cells (Fig. 2c), suggesting the regulatory role of LSH in GINS4 expression in cis. Consistent with our previous findings, we found that LSH was highly expressed in lung cancer tissues (Fig. 2d) and was positively correlated with GINS4 expression (Fig. 2e).

Chromatin modifiers might interact with both chromatin and mRNA transcripts [9-11], indicating that chromatin modifiers are involved in the post-transcriptional regulation. To determine whether LSH protein binds to GINS4 mRNA, we performed RNA immunoprecipitation (RIP) assay by using an anti-LSH antibody. We found that LSH was recruited to the GINS4 mRNA in PC9-LSH and $\mathrm{H} 358$-LSH cells but was not recruited to the intron region of the GINS4 mRNA (Fig. 2f-g).

Next, we constructed a series of vectors expressing truncated LSH fragments (LSH fragment lacking the Nterminal domain and containing a coiled-coil domain [1-226 AA], LSH fragment containing an ATP-binding 
Table 1 GINS4 expression level and Main characteristics of the patients ( $N=79)$

\begin{tabular}{|c|c|c|c|}
\hline Characteristics & $n(\%)$ & Relative expression level (Mean) & $P$ value \\
\hline \multicolumn{4}{|l|}{ Gender } \\
\hline Male & $55(70)$ & 0.024 & \multirow[t]{2}{*}{0.273} \\
\hline Female & $24(30)$ & 0.027 & \\
\hline \multicolumn{4}{|l|}{ Smoking History } \\
\hline Yes & $41(52)$ & 0.028 & \multirow[t]{2}{*}{0.280} \\
\hline No & $38(48)$ & 0.024 & \\
\hline \multicolumn{4}{|l|}{ Histology } \\
\hline ADC & $49(62)$ & 0.028 & \multirow[t]{2}{*}{0.359} \\
\hline SCC & $30(38)$ & 0.023 & \\
\hline \multicolumn{4}{|l|}{ Differentiation } \\
\hline Poor and moderate & $70(89)$ & 0.029 & \multirow[t]{2}{*}{0.115} \\
\hline Well & $9(11)$ & & \\
\hline \multicolumn{4}{|l|}{ T stage } \\
\hline T1 & $18(23)$ & 0.021 & \multirow[t]{3}{*}{0.004} \\
\hline $\mathrm{T} 2$ & $59(75)$ & 0.029 & \\
\hline T3 & $5(6)$ & 0.041 & \\
\hline \multicolumn{4}{|l|}{ N stage } \\
\hline NO & $44(56)$ & 0.020 & \multirow[t]{2}{*}{0.002} \\
\hline N1-N3 & $35(44)$ & 0.033 & \\
\hline \multicolumn{4}{|l|}{ Clinical Stages } \\
\hline$|-| \mid$ & $58(73)$ & 0.023 & \multirow[t]{2}{*}{$<0.001$} \\
\hline III-IV & $21(27)$ & 0.036 & \\
\hline
\end{tabular}

domain [227-589 AA], and LSH fragment containing Cterminal region of SNF2 domain [590-838 AA]; Fig. 2h). Analysis by using a series of truncated LSH fragments showed that both 1-226 AA and 227-589 AA of LSH interacted with GINS4 mRNA (Fig. 2i), indicating that LSH binding directly induced changes in GINS4 mRNA expression and that the ATP-binding domain of LSH was mainly critical for the binding of LSH to GINS4 mRNA.

LSH increases GINS4 stabilization through 3'UTR of GINS4 The treatment of LSH-overexpressing cells with a transcriptional inhibitor actinomycin D [37] showed that LSH overexpression significantly increased the half-life of GINS4 mRNA by 2.4 fold but did not affect the stability of $\beta$-actin mRNA in PC9 cells (Fig. 3a). LSH overexpression also significantly increased the half-life of GINS4 mRNA by two fold but did not affect the stability of $\beta$-actin mRNA in H358 cells (Fig. 3b). However, LSH depletion significantly decreased the half-life of GINS4 mRNA by two fold in A549 cells (Fig. 3c). Results of reporter assays showed an increase in luciferase activity in 293 cells coexpressing full-length GINS4 3' UTR (Luc + 3'UTR) and LSH (Fig. 3d). This may be because of an increase in GINS4's regulation of LSH through its 3'UTR. This regulatory induction was decreased after LSH depletion in A549 cells (Fig. 3e). Together, these findings indicate that LSH increases GINS4 expression and stabilization through directly binding.

\section{Overexpression of GINS4 promotes cancer cell growth, migration and invasion}

To address the role of GINS4 in lung cancer, we first performed western blotting analysis to determine GINS4 expression in a panel of lung cells and found that GINS4 expression was higher in lung cancer cells than in normal lung cells (Additional file 1: Figure S2A). Results of qRTPCR confirmed that GINS4 expression was higher in lung cancer cells than in normal lung cells (Additional file 1: Figure S2B). Western blotting analysis of the nuclear and cytoplasmic fractions of H1299, 95C, PC9, A549, SPCA-1 and HBE cells showed that majority of GINS4 was present in the nucleus of these cells (Additional file 1: Figure S2C). Next, we selected HBE, A549, PC9, and H1299 cells to determine the role of GINS4 in lung cancer progression.

We first stably overexpressed GINS4 in lung cancer cell lines PC9 and H358, and we also found that GINS4 did not affect LSH expression (PC9-GINS4 and H358GINS4, respectively; Additional file 1: Figure S2D). 


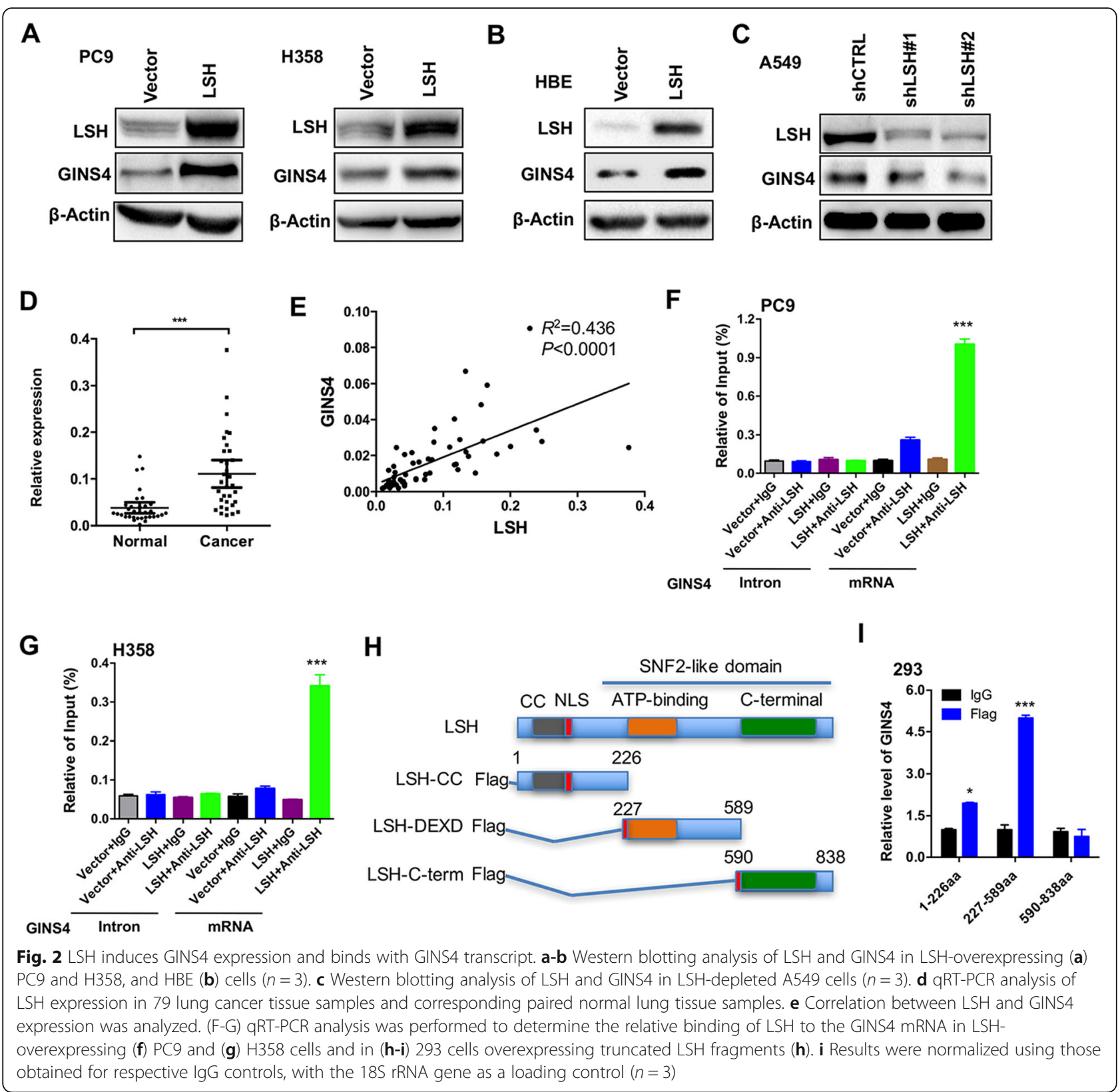

GINS4 overexpression significantly increased the growth of these cells in vitro (Fig. 4a-b) and enhanced their colony formation ability (Fig. 4c-d). Moreover, stable GINS4 expression increased the migration and invasion of PC9 and H358 cells in vitro (Fig. 4e-f). Furthermore, stable GINS4 expression impaired the expression of epithelial marker E-cadherin in PC9 cells and in H358 cells and increased the expression of mesenchymal markers Vimentin and Snail (Fig. 4g-h), suggesting that GINS4 promoted epithelial-mesenchymal transition (EMT) in these cells. Analysis by using the high-content imaging system showed that GINS4 overexpression decreased the relative intensity of E-cadherin staining and increased the relative intensity of vimentin staining in both PC9 and H358 cells (Fig. 4i). Next, we found that the GINS4 expressing cells formed larger and more abundant tumor spheres that the control cells in both PC9 and H358 cells through tumor sphere assays (both ** $P<0.01$ ) (Fig. 4j).

To determine whether GINS4 also played a role in lung cancer progression in vivo, we established a xenograft nude mouse model and examined xenograft tumor formation in this model. We found that nude mice injected with PC9-GINS4 cells formed tumors of significantly larger tumor volume, tumor size and tumor weight than nude mice injected with control cells (Fig. $4 \mathrm{k}-\mathrm{m}$ ). The injection of H358-GINS4 cells $\left(1 \times 10^{7}\right)$ also showed that 


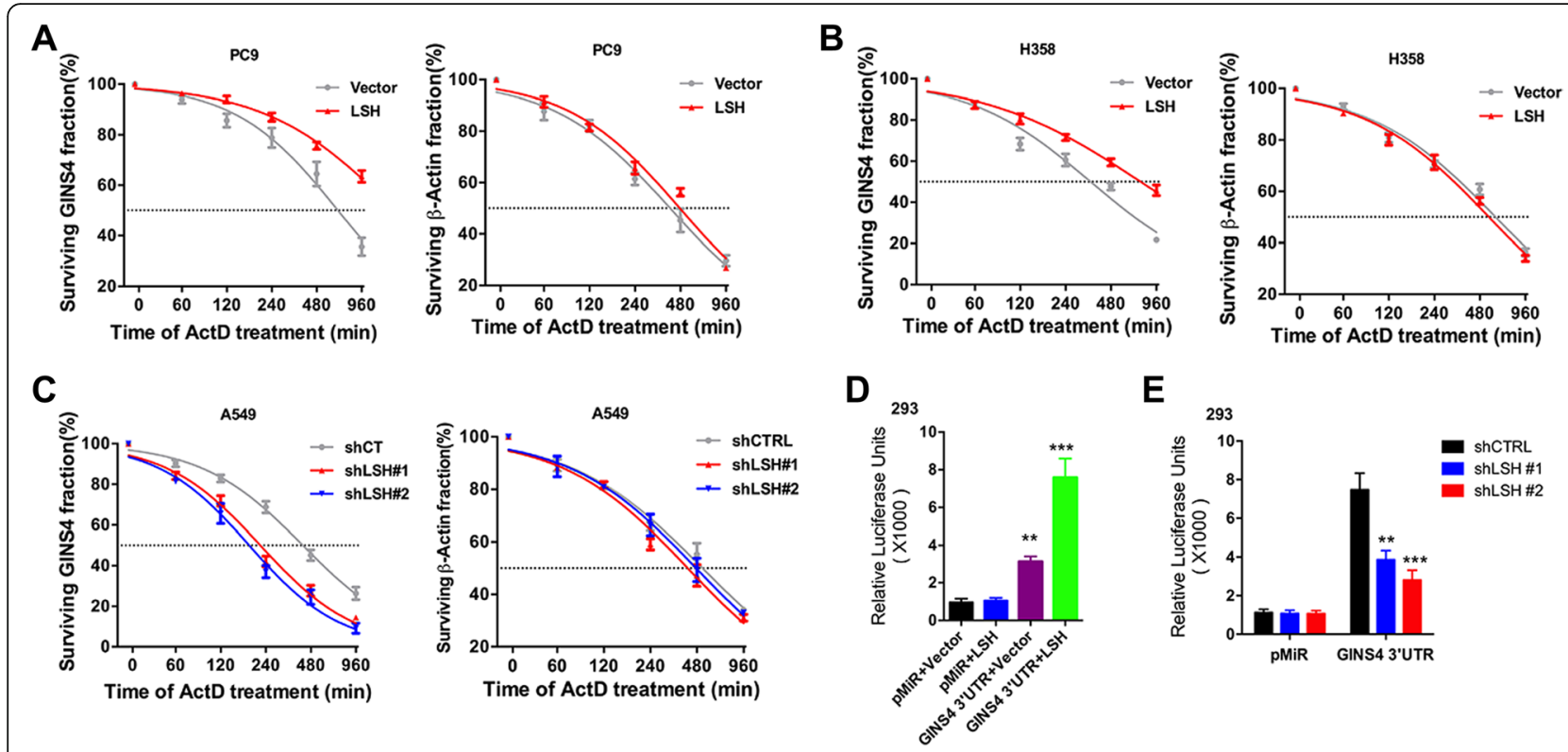

Fig. 3 LSH increases GINS4 stabilization through 3'UTR of GINS4. a-c LSH-overexpressing (a) PC9 and (b) H358 cells and (c) LSH-knockout A549 cells were treated with actinomycin D $(5 \mu \mathrm{g} / \mathrm{mL})$ for the indicated time. G/NS4 and $\beta$-actin mRNA stability was assayed by performing qRT-PCR, with the 185 rRNA gene as a loading control $(n=3)$. $\mathbf{d}$ Luciferase activity in 293 cells coexpressing a luciferase reporter vector encoding the GINS4 $3^{\prime} \mathrm{UTR}$ and LSH ( $\left.n=4\right)$. e Luciferase activity in LSH-depleted A549 cells expressing a luciferase reporter vector encoding the GINS4 $3^{\prime}$ UTR $(n=4)$. ${ }^{*} P<0.05$, ${ }^{* *} P<0.01$, and ${ }^{* * *} P<0.001$

GIINS4 overexpression significantly increased the tumor volume, tumor size, and tumor weight (Fig. 4n-p). Furthermore, GINS4 accelerated EMT through Vimentin and E-cadherin staining after overexpression of GINS4 in PC9 and H358 biopsies compared with that in the control cells biopsies from xenograft tumors. However, GINS4 did not change LSH expression according to IHC in LSH in PC9 and H358 cells biopsies among the three biopsies (Additional file 1: Figure S3). Together, these results indicate that GINS4 overexpression promotes the growth, migration, and invasion of lung cancer cells.

\section{GINS4 knockdown inhibits cancer progression in vitro}

To further validate the role of GINS4 in lung cancer progression, we generated stable GINS4- knockdown H1299 cells. All GINS4-targeting shRNAs reduced GINS4 mRNA and protein levels in H1299 cells; moreover, GINS4 knockdown by using shGINS4\#1 and shGINS4\#2 successfully reduced GINS4 mRNA and protein levels to $<10 \%$ (Fig. 5a-b). GINS4 knockdown significantly reduced the growth of H1299 (Fig. 5c). Moreover, GINS4 knockdown impaired the colony formation ability (Fig. 5d) and reduced the migration and invasion abilities of H1299 cells (Fig. 5e). Moreover, stable GINS4 knockdown increased the relative intensity of E-cadherin and impaired the relative intensity of Vimentin and Snail staining in H1299 cells (Fig. 5f). Analysis by using the high-content imaging system showed that GINS4 knockdown increased E-cadherin staining and attenuated vimentin expression in H1299 cells (Fig. 5g). Next, we demonstrated that depletion of GINS4 formed smaller and less abundant tumor spheres that the control cells in H1299 cells through tumor sphere assays (both * ${ }^{*}<0.01$ ) (Fig. 5h). Together, these results indicate that depletion of GINS4 inhibits the growth, migration, and invasion of lung cancer cells.

\section{GINS4 knockdown inhibits cancer progression in vivo}

To further determine whether GINS4 also played a role in lung cancer progression in vivo, we injected $3 \times 10^{6}$ GINS4- knockdown H1299 cells into nude mice and found that GINS4 knockdown significantly decreased the volume, size and weight of tumors derived from these cells (Fig. 6a-c). However, no significant change was observed in the body weights of mice injected with GINS4-knockdown H1299 cells and control cells. Furthermore, depletion of GINS4 impaired EMT through Vimentin and E-cadherin staining after depletion of GINS4 in H1299 biopsies compared with that in the control cells biopsies from xenograft tumors. However, GINS4 did not change LSH expression in H1299 cells after depletion of GINS4 biopsies among the three biopsies (Fig. 6d). Together, these findings indicate the physiological role of GINS4 in the growth, migration, and invasion characteristics of lung cancer cells.

To further address whether GINS4 promotes tumorigenic potential under the control of LSH, we detected cell growth of lung cancer cells after LSH was transfected into the GINS4-depleted cells. We found that LSH overexpression-induced growth proliferation of H1299 


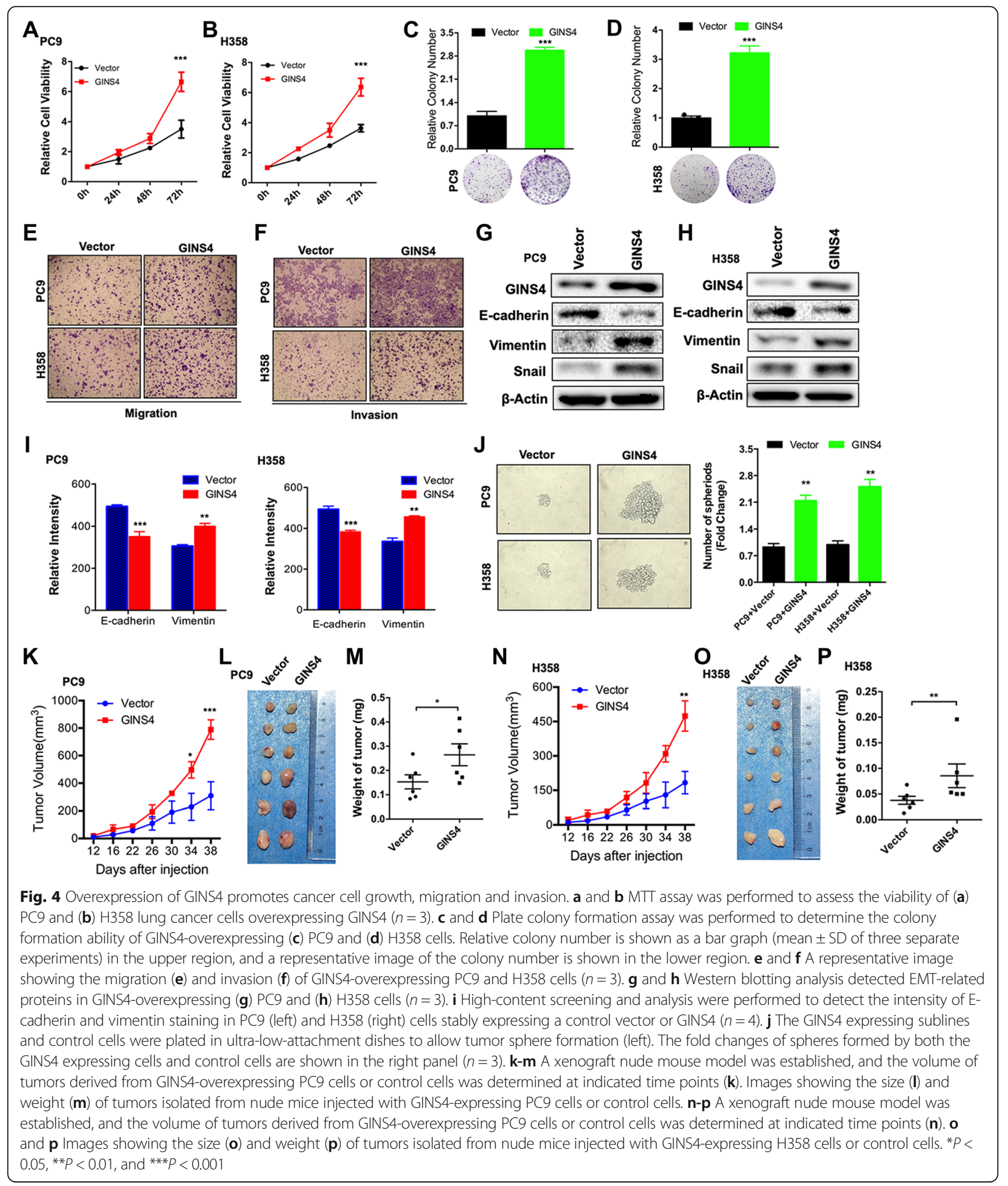

cells was attenuated after depletion of GINS4 (Fig. 6e). Moreover, we showed that the increase of LSH in migration and invasion abilities of H1299 cells was attenuated after depletion of GINS4. Taken together, GINS4 accelerates tumorigenic potential dependent of LSH expression.

\section{Discussion}

In the present study, we found that GINS4 facilitates lung cancer progression by promoting cancer cell growth, migration, and invasion, which are the key characteristics of cancer progression. Epigenetic regulation 


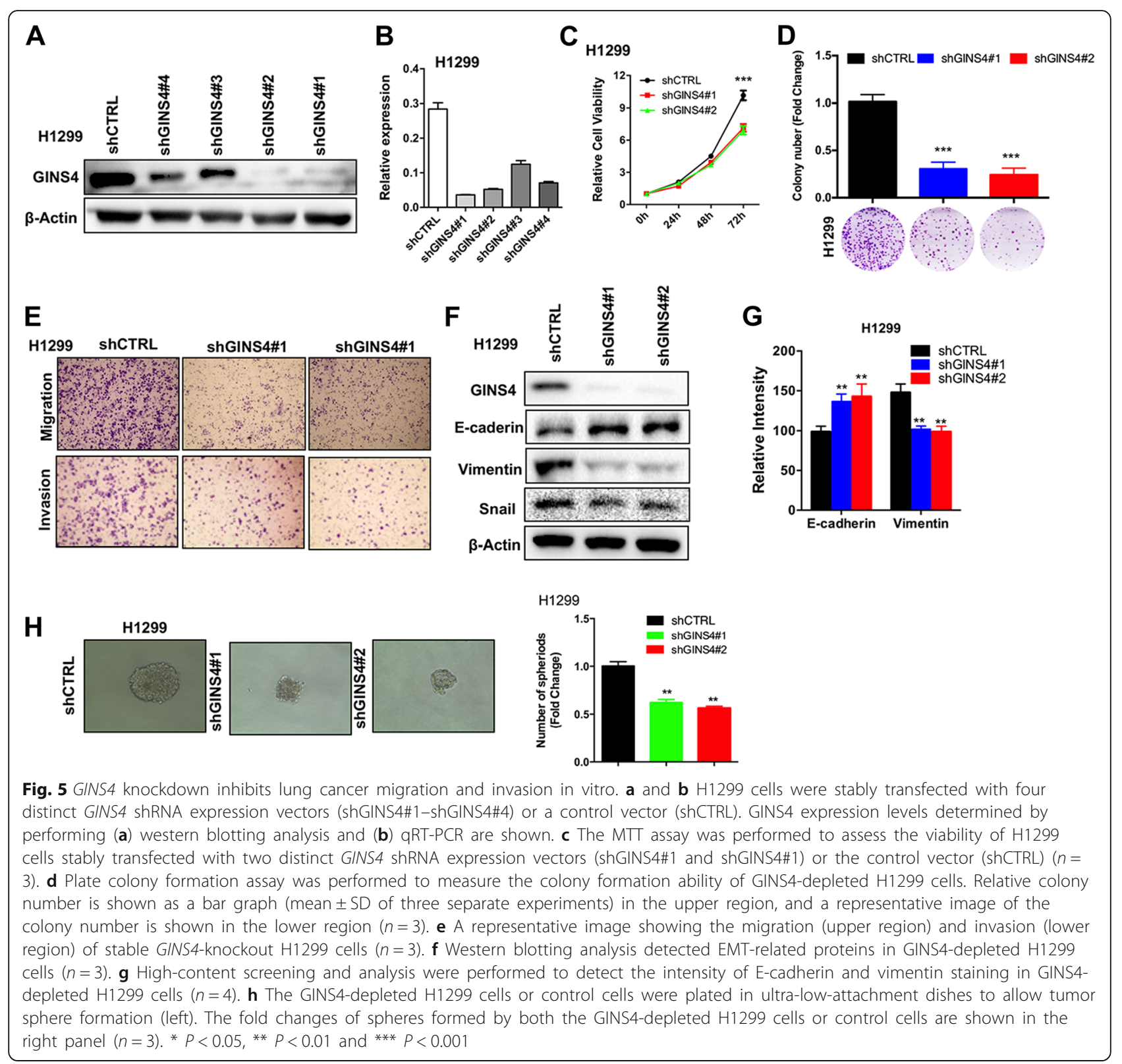

involves in the regulation of GINS4, LSH upregulates GINS4 by stabilizing GINS4 mRNA level.

LSH is critical for DNA methylation because it functions as a chromatin silencer [16, 17, 38, 39]. Moreover, LSH increases nucleosome density [39], induces RNA polymerase II stalling $[40,41]$ and promotes gene silencing through a G9a-GLP complex during differentiation and early development [18]. Moreover, LSH contributes to carcinogenesis as a transcriptional repressor [22, 23, 25, 36, 42, 43]. Interestingly, we found that LSH overexpression upregulated GINS4 expression independently of DNA methylation. We further firstly provide evidence for the increased role of LSH in gene expression through mRNA stability instead of silencing, indicating that LSH involves the target genes in a selective manner. Our findings further indicate that chromatin modifiers, including histone methyltransferases, post-transcriptionally regulate mRNA levels [9-11, 44].

GINS4 complexes with other moieties such as PSF1, PSF2, and PSF3 to promote DNA replication in yeast [27, $28,45]$. High expression of GINS genes such as PSF1 has been detected in different cancers, and expression levels of GINS genes are suggested to be correlated with growth retardation, cell cycle arrest, malignancy, and stem celllike properties; for example, high PSF1 promoter activity has been detected in cancer-initiating cells or cancer stem cells in a murine tumor cell transplantation model 


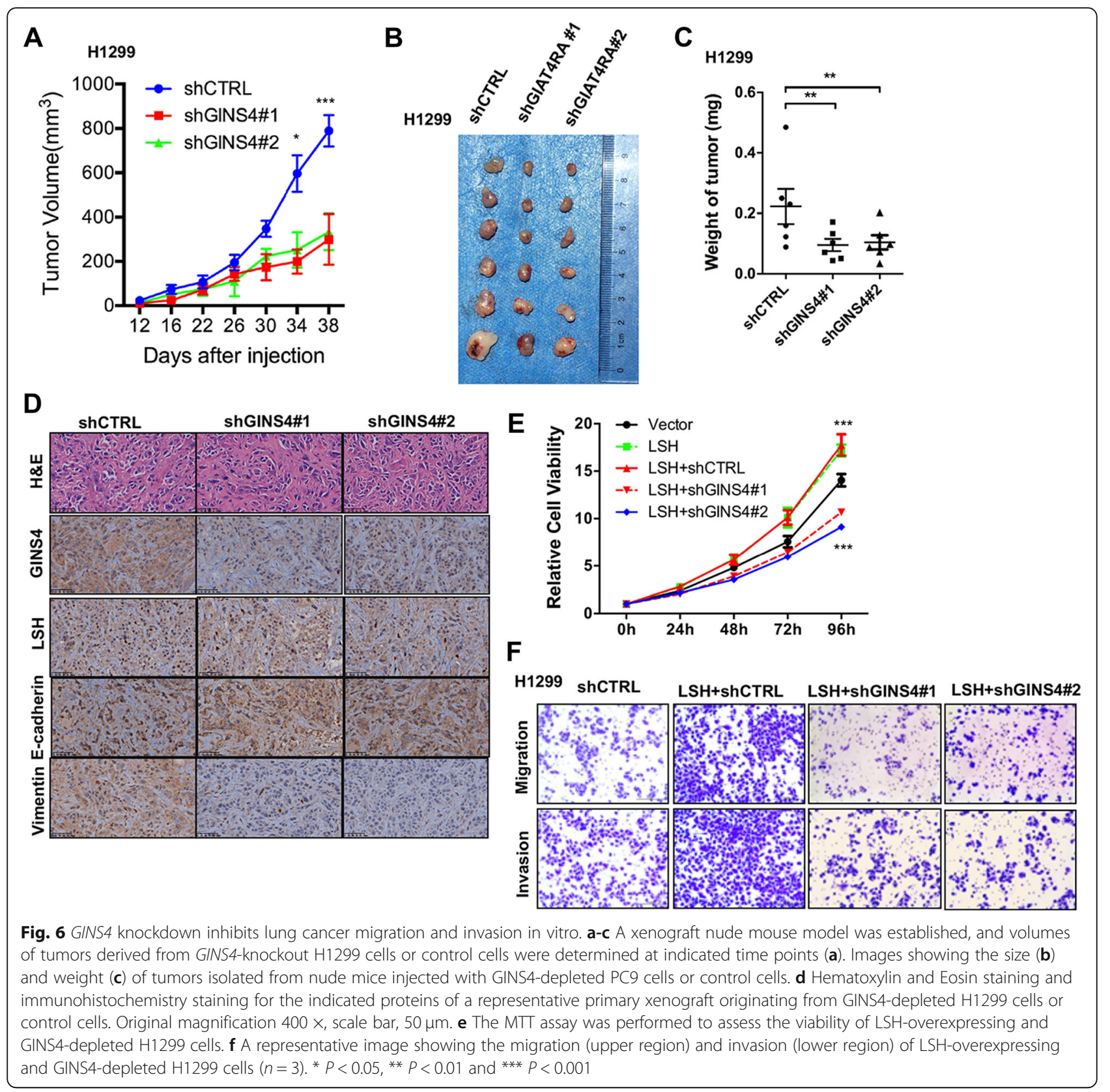

[46, 47]. High expression levels of GINS genes may induce cell growth as well as chemotherapy resistance. Interestingly, another replication licensing factor Cdc6 undergoes phenotypic changes with mesenchymal features and loss of E-cadherin by direct binding to the promoter beyond activating adjacent replication origins [48]. In the present study, we analyzed the functions of GINS4 besides DNA replication and found that GINS4 promoted EMT, migration, invasion, and metastasis in lung cancer. High expression levels of PSF1 and PSF2 but not of PSF3 are associated with the poor survival of patients with lung cancer and ADC but not the survival of patients with SCC (Additional file 1: Figure S4). The reasons for this is possible that the driver genes in SCCs and ADCs are different [49].

Interestingly, high expression of GINS4 is associated with the poor survival of patients with lung ADC and gastric cancer, but not the survival of patients with lung SCC and breast cancer (Additional file 1: Figure S5), indicating that GISN4 functions as an oncogene depending on the cancer type.

\section{Conclusions}

In summary, our study highlights the importance of GINS4 in lung cancer migration, invasion, and progression. LSH increased GINS4 protein level by increasing the 
stability of the GINS4 mRNA. GINS4 as well as other GINS family members have the potential to regulate cancer cell proliferation, suggesting their use as therapeutic targets for cancer treatment. Our findings suggest that the LSH-GINS4 axis can be used as a potential target for developing novel therapeutic approaches.

\section{Additional files}

Additional file 1: Expression of GINS4 in various malignant tumors and its relationship with prognosis. (DOC $13705 \mathrm{~kb}$ )

\section{Abbreviations}

3'UTR:: 3'-untranslated region; ADC: Adenocarcinomas; Co-IP: CoImmunoprecipitation; EMT: Epithelial-mesenchymal transition; GINS4: Go, Ichi, Nii, and San (means five, one, two, and three, respectively, in Japanese) complex subunit 4; IHC: Immunohistochemistry; LSH: Lymphoid-specific helicase; NSCLC: Non-small cell lung cancer; RIP: RNA immunoprecipitation; SCC: Sequamous cell carcinoma

\section{Acknowledgements}

We thank Prof. Dangsheng Li (Deputy Editor-in-Chief, Cell Research, Shanghai Institutes for Biological Sciences, Chinese Academy of Sciences, Shanghai 200031 China) and Prof. Teibang Kang (State Key Laboratory of Oncology in South China, Collaborative Innovation Center for Cancer Medicine, Sun Yat-sen University Cancer Center, No. 651 Dongfeng East Road, Guangzhou, 510060, China) very much for the kind suggestions. We also thank Prof. Jian Ma (Cancer Research Institute, Central South University, 110 Xiangya Road, Changsha, Hunan, 410078 China) very much for the kind help including providing cell lines.

\section{Authors' contributions}

In this report, YT, QY, and SL designed the experiments and drafted the manuscript; RY, NL, LC, YJ, YS, YL, CM, MW, WL, HT and MG performed the experiments. DX, XW and SL were responsible for sample collection and data analysis; SL, WL, FY, YC, QY and YT discussed and revised the manuscript. YT, QY and SL was the originator of the concept of this report and wrote and approved the manuscript. All of the authors approved this manuscript.

\section{Funding}

This work was supported by the National Natural Science Foundation of China (81,672,787 and 81,728,014[Y. Tao], 81,672,991 and 81,874,139 [S. Liu], $81,672,307$ [X. Wang]), the National Basic Research Program of China (2015CB553903 [Y. Tao]), and the Fundamental Research Funds for the Central Universities (2017zzts828 [R. Yang] and 2017zzts206 [N. Liu]).

\section{Availability of data and materials}

Not applicable.

\section{Ethics approval and consent to participate}

This study was conducted at the Cancer Research Institute, Central South University, Hunan, China. All of the protocols were reviewed and approved by the Joint Ethics Committee of the Central South University Health Authority and performed in accordance with national guidelines.

\section{Consent for publication}

This manuscript has been read and approved by all the authors to publish and is not submitted or under consideration for publication elsewhere.

\section{Competing interests}

The authors declare that they do not have any conflicts of interest related to this study. This manuscript has been read and approved by all the authors and has not been submitted to or is not under consider for publication elsewhere.

\section{Author details}

'Department of Pathology, Key Laboratory of Carcinogenesis and Cancer Invasion, Ministry of Education, Xiangya Hospital, Central South University, Changsha 410078, Hunan, China. ${ }^{2} \mathrm{NHC}$ Key Laboratory of Carcinogenesis of
Ministry of Health (Central South University), Cancer Research Institute; School of Basic Medicine, Central South University, Changsha 410078, Hunan, China. ${ }^{3}$ Department of Pathology, Xiangya Hospital, Central South University, Changsha 410008, Hunan, China. ${ }^{4}$ Department of Thoracic Surgery, Second Xiangya Hospital, Central South University, Changsha, China. ${ }^{5}$ Department of Pathology, Yale School of Medicine, New Haven, CT 06520, USA.

${ }^{6}$ Department of Oncology, Institute of Medical Sciences, Xiangya Hospital, Central South University, Changsha 410008, Hunan, China.

Received: 9 March 2019 Accepted: 11 June 2019

Published online: 28 June 2019

\section{References}

1. Reck M, Heigener DF, Mok T, Soria JC, Rabe KF. Management of non-smallcell lung cancer: recent developments. Lancet. 2013;382:709-19.

2. Chen W, Zheng R, Baade PD, Zhang S, Zeng H, Bray F, Jemal A, Yu XQ, He J. Cancer statistics in China, 2015. CA Cancer J Clin. 2016;66:115-32.

3. Forde PM, Brahmer JR, Kelly RJ. New strategies in lung cancer: epigenetic therapy for non-small cell lung cancer. Clin Cancer Res. 2014;20:2244-8.

4. Feinberg AP, Koldobskiy MA, Gondor A. Epigenetic modulators, modifiers and mediators in cancer aetiology and progression. Nat Rev Genet. 2016;17:284-99.

5. Bhan A, Soleimani M, Mandal SS. Long noncoding RNA and Cancer: a new paradigm. Cancer Res. 2017;77:3965-81.

6. Batista PJ, Chang HY. Long noncoding RNAs: cellular address codes in development and disease. Cell. 2013;152:1298-307.

7. Schmitt AM, Chang HY. Long noncoding RNAs in Cancer pathways. Cancer Cell. 2016;29:452-63.

8. White NM, Cabanski CR, Silva-Fisher JM, Dang HX, Govindan R, Maher CA. Transcriptome sequencing reveals altered long intergenic non-coding RNAs in lung cancer. Genome Biol. 2014;15:429.

9. Blair LP, Liu Z, Labitigan RL, Wu L, Zheng D, Xia Z, Pearson EL, Nazeer FI, Cao J, Lang SM, et al. KDM5 lysine demethylases are involved in maintenance of 3'UTR length. Sci Adv. 2016;2:e1501662.

10. Chand SN, Zarei M, Schiewer MJ, Kamath AR, Romeo C, Lal S, Cozzitorto JA, Nevler A, Scolaro L, Londin E, et al. Post-transcriptional regulation of PARG mRNA by HuR facilitates DNA repair and resistance to PARP inhibitors. Cancer Res. 2017:77:5011-25.

11. Zarei M, Lal S, Parker SJ, Nevler A, Vaziri-Gohar A, Dukleska K, MambelliLisboa NC, Moffat C, Blanco FF, Chand SN, et al. Posttranscriptional upregulation of IDH1 by HuR establishes a powerful survival phenotype in pancreatic Cancer cells. Cancer Res. 2017:77:4460-71.

12. Raab JR, Smith KN, Spear CC, Manner CJ, Calabrese JM, Magnuson T. SWI/ SNF remains localized to chromatin in the presence of SCHLAP1. Nat Genet 2018;51:26-9.

13. Mashtalir N, D'Avino AR, Michel BC, Luo J, Pan J, Otto JE, Zullow HJ, McKenzie ZM, Kubiak RL, St Pierre R, et al. Modular organization and assembly of SWI/SNF family chromatin remodeling complexes. Cell. 2018; 175:1272-1288 e1220.

14. Kadoch C, Crabtree GR. Mammalian SWI/SNF chromatin remodeling complexes and cancer: mechanistic insights gained from human genomics. Sci Adv. 2015;1:e1500447.

15. Zemach A, Kim MY, Hsieh PH, Coleman-Derr D, Eshed-Williams L, Thao K, Harmer SL, Zilberman D. The Arabidopsis nucleosome remodeler DDM1 allows DNA methyltransferases to access $\mathrm{H1}$-containing heterochromatin. Cell. 2013;153:193-205

16. Yu W, McIntosh C, Lister R, Zhu I, Han Y, Ren J, Landsman D, Lee E, Briones $V$, Terashima M, et al. Genome-wide DNA methylation patterns in LSH mutant reveals de-repression of repeat elements and redundant epigenetic silencing pathways. Genome Res. 2014b;24:1613-23.

17. Tao Y, Xi S, Shan J, Maunakea A, Che A, Briones V, Lee EY, Geiman T, Huang J, Stephens R, et al. Lsh, chromatin remodeling family member, modulates genome-wide cytosine methylation patterns at nonrepeat sequences. Proc Natl Acad Sci U S A. 2011b;108:5626-31.

18. Myant K, Termanis A, Sundaram AY, Boe T, Li C, Merusi C, Burrage J, de Las Heras II, Stancheva I. LSH and G9a/GLP complex are required for developmentally programmed DNA methylation. Genome Res. 2011;21: 83-94.

19. Dunican DS, Cruickshanks HA, Suzuki M, Semple CA, Davey T, Arceci RJ, Greally J, Adams IR, Meehan RR. Lsh regulates LTR retrotransposon repression independently of Dnmt3b function. Genome Biol. 2013;14:R146. 
20. Fan T, Yan Q, Huang J, Austin S, Cho E, Ferris D, Muegge K. Lsh-deficient murine embryonal fibroblasts show reduced proliferation with signs of abnormal mitosis. Cancer Res. 2003;63:4677-83.

21. Burrage J, Termanis A, Geissner A, Myant K, Gordon K, Stancheva I. The SNF2 family ATPase LSH promotes phosphorylation of H2AX and efficient repair of DNA double-strand breaks in mammalian cells. J Cell Sci. 2012;125:5524-34.

22. von Eyss B, Maaskola J, Memczak S, Mollmann K, Schuetz A, Loddenkemper C, Tanh MD, Otto A, Muegge K, Heinemann U, et al. The SNF2-like helicase HELLS mediates E2F3-dependent transcription and cellular transformation. EMBO J. 2012;31:972-85.

23. Xiao D, Huang J, Pan Y, Li H, Fu C, Mao C, Cheng Y, Shi Y, Chen L, Jiang Y, et al. Chromatin remodeling factor LSH is upregulated by the LRP6-GSK3beta-E2F1 Axis linking reversely with survival in gliomas. Theranostics. 2017;7:132-43.

24. Keyes WM, Pecoraro M, Aranda V, Vernersson-Lindahl E, Li W, Vogel H, Guo X, Garcia EL, Michurina TV, Enikolopov G, et al. DeltaNp63alpha is an oncogene that targets chromatin remodeler Lsh to drive skin stem cell proliferation and tumorigenesis. Cell Stem Cell. 2011;8:164-76.

25. He X, Yan B, Liu S, Jia J, Lai W, Xin X, Tang CE, Luo D, Tan T, Jiang Y, et al. Chromatin remodeling factor LSH drives Cancer progression by suppressing the activity of fumarate hydratase. Cancer Res. 2016a;76:5743-55.

26. Wang R, Shi Y, Chen L, Jiang Y, Mao C, Yan B, Liu S, Shan B, Tao Y, Wang X. The ratio of FoxA1 to FoxA2 in lung adenocarcinoma is regulated by LncRNA HOTAIR and chromatin remodeling factor LSH. Sci Rep. 2015;5:17826.

27. Kong L, Ueno M, Itoh M, Yoshioka K, Takakura N. Identification and characterization of mouse PSF1-binding protein, SLD5. Biochem Biophys Res Commun. 2006;339:1204-7.

28. Kamada K, Kubota Y, Arata T, Shindo Y, Hanaoka F. Structure of the human GINS complex and its assembly and functional interface in replication initiation. Nat Struct Mol Biol. 2007;14:388-96.

29. Labib K, Gambus A. A key role for the GINS complex at DNA replication forks. Trends Cell Biol. 2007;17:271-8.

30. Shi $Y$, Tao $Y$, Jiang $Y, X u$ Y, Yan B, Chen X, Xiao L, Cao Y. Nuclear epiderma growth factor receptor interacts with transcriptional intermediary factor 2 to activate cyclin D1 gene expression triggered by the oncoprotein latent membrane protein 1. Carcinogenesis. 2012;33:1468-78.

31. Jiang Y, Yan B, Lai W, Shi Y, Xiao D, Jia J, Liu S, Li H, Lu J, Li Z, et al. Repression of Hox genes by LMP1 in nasopharyngeal carcinoma and modulation of glycolytic pathway genes by HoxC8. Oncogene. 2015;34:6079-91.

32. Mao C, Wang $X$, Liu $Y$, Wang $M$, Yan B, Jiang $Y$, Shi $Y$, Shen $Y$, Liu X, Lai W, et al. A G3BP1-interacting IncRNA promotes Ferroptosis and apoptosis in Cancer via nuclear sequestration of p53. Cancer Res. 2018a;78:3484-96.

33. Yan B, Liu S, Shi Y, Liu N, Chen L, Wang X, Xiao D, Liu X, Mao C, Jiang Y, et al. Activation of AhR with nuclear IKKalpha regulates cancer stem-like properties in the occurrence of radioresistance. Cell Death Dis. 2018;9:490.

34. Shi Y, Liu N, Lai W, Yan B, Chen L, Liu S, Liu S, Wang X, Xiao D, Liu X, et al. Nuclear EGFR-PKM2 axis induces cancer stem cell-like characteristics in irradiation-resistant cells. Cancer Lett. 2018:422:81-93.

35. Mao C, Wang M, Qian B, Ouyang L, Shi Y, Liu N, Chen L, Xiao D, Wang X, Cao $Y$, et al. Aryl hydrocarbon receptor activated by benzo (a) pyrene promotes SMARCA6 expression in NSCLC. Am J Cancer Res. 2018b;8:1214-27.

36. Jiang YMC, Yang R, Yan B, Shi Y, Liu X, Lai W, Liu Y, Wang X, Xiao D, Zhou H, Cheng Y, Yu F, Cao Y, Liu S, Yan Q, Tao Y. EGLN1/C-Myc induced lymphoid-specific helicase inhibits Ferroptosis through lipid metabolic gene expression changes. Theranostics. 2017b;7:3293-305.

37. Lal S, Burkhart RA, Beeharry N, Bhattacharjee V, Londin ER, Cozzitorto JA, Romeo C, Jimbo M, Norris ZA, Yeo CJ, et al. HuR posttranscriptionally regulates WEE1: implications for the DNA damage response in pancreatic cancer cells. Cancer Res. 2014;74:1128-40.

38. Yu W, Briones V, Lister R, McIntosh C, Han Y, Lee EY, Ren J, Terashima M Leighty RM, Ecker JR, Muegge K. CG hypomethylation in Lsh-/- mouse embryonic fibroblasts is associated with de novo H3K4me1 formation and altered cellular plasticity. Proc Natl Acad Sci U S A. 2014a;111:5890-5.

39. Ren J, Briones V, Barbour S, Yu W, Han Y, Terashima M, Muegge K. The ATP binding site of the chromatin remodeling homolog $L$ sh is required for nucleosome density and de novo DNA methylation at repeat sequences. Nucleic Acids Res. 2015:43:1444-55.

40. Tao Y, Liu S, Briones V, Geiman TM, Muegge K. Treatment of breast cancer cells with DNA demethylating agents leads to a release of pol II stalling at genes with DNA-hypermethylated regions upstream of TSS. Nucleic Acids Res. 2011a;39:9508-20.
41. Tao Y, Xi S, Briones V, Muegge K. Lsh mediated RNA polymerase II stalling at HoxC6 and HoxC8 involves DNA methylation. PLoS One. 2010;5:e9163.

42. Jia JSY, Chen L, Lai W, Yan B, Jiang Y, Xiao D, Xi S, Cao Y, Liu S, Cheng Y, Tao Y. Decrease in lymphoid specific helicase and 5-hydroxymethylcytosine is associated with metastasis and genome instability. Theranostics. 2017;7:3920-32.

43. Liu S, Tao YG. Chromatin remodeling factor LSH affects fumarate hydratase as a cancer driver. Chin J Cancer. 2016;35:72

44. Sayou C, Millan-Zambrano G, Santos-Rosa H, Petfalski E, Robson S, Houseley J, Kouzarides T, Tollervey D. RNA Binding by Histone Methyltransferases Set1 and Set2. Mol Cell Biol. 2017;37:e00165-17.

45. Looke M, Maloney MF, Bell SP. Mcm10 regulates DNA replication elongation by stimulating the CMG replicative helicase. Genes Dev. 2017;31:291-305.

46. Gong ZY, Kidoya H, Mohri T, Han Y, Takakura N. DNA damage enhanced by the attenuation of SLD5 delays cell cycle restoration in normal cells but not in cancer cells. PLoS One. 2014;9:e110483.

47. Yamane K, Naito H, Wakabayashi T, Yoshida H, Muramatsu F, Iba T, Kidoya H, Takakura N. Regulation of SLD5 gene expression by miR-370 during acute growth of cancer cells. Sci Rep. 2016;6:30941.

48. Sideridou M, Zakopoulou R, Evangelou K, Liontos M, Kotsinas A, Rampakakis E, Gagos S, Kahata K, Grabusic K, Gkouskou K, et al. Cdc6 expression represses $\mathrm{E}$-cadherin transcription and activates adjacent replication origins. J Cell Biol. 2011:195:1123-40.

49. Campbell JD, Alexandrov A, Kim J, Wala J, Berger AH, Pedamallu CS, Shukla SA, Guo G, Brooks AN, Murray BA, et al. Distinct patterns of somatic genome alterations in lung adenocarcinomas and squamous cell carcinomas. Nat Genet. 2016:48:607-16.

\section{Publisher's Note}

Springer Nature remains neutral with regard to jurisdictional claims in published maps and institutional affiliations.

Ready to submit your research? Choose BMC and benefit from:

- fast, convenient online submission

- thorough peer review by experienced researchers in your field

- rapid publication on acceptance

- support for research data, including large and complex data types

- gold Open Access which fosters wider collaboration and increased citations

- maximum visibility for your research: over $100 \mathrm{M}$ website views per year

At $\mathrm{BMC}$, research is always in progress.

Learn more biomedcentral.com/submission 